\title{
Radionuclide geochemistry: solubility and thermodynamics in an HLW repository
}

\author{
David Fellhauer, Xavier Gaona, Marcus Altmaier, and Horst Geckeis \\ Institut für Nukleare Entsorgung (INE), Karlsruher Institut für Technologie (KIT), 76131 Karlsruhe, Germany \\ Correspondence: David Fellhauer (david.fellhauer@kit.edu) \\ Published: 10 November 2021
}

\begin{abstract}
Deep geological disposal is the internationally favoured option to isolate high-level nuclear waste (HLW) from the biosphere and to minimise the potential radiological risk for future generations. Potentially contacting aqueous solutions such as groundwater may, however, lead to the corrosion of the solid casks containing the nuclear waste, and the formation of aqueous radionuclide systems in the near-field of the emplacement rooms. As dissolved species, radionuclides can in principle further migrate into the far-field and finally reach the biosphere on medium and long timescales. Like all chemical species, the radionuclides are subject to fundamental (geo)chemical laws. Relevant reactions that control retention and release, and hence, the migration behaviour and fate of radionuclides in a repository, are solubility equilibria, formation of soluble complexes, redox reactions, sorption on and incorporation into mineral surfaces, transport phenomena etc. These processes depend directly on the (geo)chemical boundary conditions, and, consequently, can differ greatly for various host rock systems such as clay rock, rock salt, and crystalline rock.

Many of the radionuclides in HLW are heavy metals that are sparingly soluble under various repositoryrelevant conditions, e.g. actinides, lanthanides, transition metals, so that only partial dissolution (mobilisation) from the solid waste matrices is expected. This underlines the importance of evaluating the radionuclide solubility within a geochemically based safety assessment for repositories as it provides reliable upper-limit concentrations of the mobile, potentially migrating radionuclide fraction in the near-field. In this contribution, we discuss relevant aspects related to the topic radionuclide solubility and thermodynamics in a HLW repository. This includes a summary of recent laboratory studies on the solubility behaviour and speciation of key radionuclides in repository-relevant solutions, which are an important basis for obtaining (geo)chemical information and models, and the corresponding fundamental thermodynamic constants on aqueous radionuclide systems. National and international thermodynamic database projects, where quality-assured thermodynamic data (solubility products, complex formation constants, and ion-interaction parameters) are evaluated and compiled, e.g. the Nuclear Energy Agency Thermochemical Database (http://www.oecd-nea.org, last access: 1 November 2021) or the Thermodynamic Reference Database (http://www.thereda.de, last access: 1 November 2021), are highlighted and the main remaining uncertainties discussed. The experimental information and the quantitative thermodynamic data are applied within a generic case study to demonstrate the impact of different geochemical solution conditions representing different host rock systems considered as HLW repositories in Germany on the solubility and speciation of selected radionuclides.
\end{abstract}

Kurzfassung. Geologische Tiefenlagerung ist die international bevorzugte Variante, hochradioaktiven Abfall (HAW) von der Biosphäre zu isolieren und ein potenziell radiologisches Risiko für künftige Generationen zu minimieren. Möglicher Kontakt mit wässrigen Lösungen wie dem Grundwasser kann jedoch zur Korrosion der Behälter, die die nuklearen Abfälle enthalten, und zur Bildung von wässrigen Radionuklidsystemen im Nahfeld der Einlagerungskammern führen. Radionuklide können als gelöste Spezies grundsätzlich weiter in das Fernfeld wandern und schließlich mittel- oder langfristig die Biosphäre erreichen. Weiterhin unterliegen sie wie alle chemischen Spezies den grundlegenden (geo-)chemischen Gesetzen. Relevante Reaktionen, die die Rückhaltung bzw. Freisetzung und damit das Migrationsverhalten bzw. den Verbleib von Radionukliden in einem 
Endlager steuern, sind Löslichkeitsgleichgewichte, die Bildung löslicher Komplexe, Redoxreaktionen, die Sorption an bzw. der Einbau in mineralische Oberflächen, Transportphänomene usw. Diese Vorgänge hängen direkt von den (geo-)chemischen Randbedingungen ab und können folglich für verschiedene Wirtsgesteinsysteme wie Tongestein, Steinsalz und kristallines Gestein sehr unterschiedlich ausfallen. Bei vielen der Radionuklide in HAW handelt es sich um Schwermetalle, die unter verschiedenen endlagerrelevanten Bedingungen schwer löslich sind, z. B. Aktinide, Lanthanoide oder Übergangsmetalle, so dass nur eine teilweise Auflösung (Mobilisierung) aus der festen Abfallmatrix zu erwarten ist. Dies unterstreicht die Bedeutung, die der Beurteilung der Radionuklidlöslichkeit in einer Endlagersicherheitsbewertung auf geochemischer Basis zukommt, da diese zuverlässige Obergrenzen für die Konzentrationen der mobilen, potenziell migrierenden Radionuklidfraktion im Nahfeld liefert. In diesem Beitrag erörtern wir die relevanten, mit der Löslichkeit und Thermodynamik von Radionukliden zusammenhängenden Aspekte in einem HAW-Endlager. Dies beinhaltet eine Zusammenfassung aktueller Laborstudien zum Löslichkeitsverhalten und zur Speziation der wichtigsten Radionuklide in endlagerrelevanten Lösungen, die eine wesentliche Grundlage für die Gewinnung (geo)chemischer Informationen und Modelle sowie der entsprechenden thermodynamischen Konstanten für wässrige Radionuklidsysteme bilden. Nationale und internationale thermodynamische Datenbankprojekte, in denen qualitätsgesicherte thermodynamische Daten (Löslichkeitsprodukte, Komplexbildungskonstanten und Ion-Wechselwirkungsparameter) ausgewertet und zusammengestellt werden, z. B. NEA-TDB (http://www.oecd-nea.org, online aufrufbar: 1 November 2021) oder THEREDA (http://www. thereda.de, online aufrufbar: 1 November 2021), werden behandelt und die wesentlichen verbleibenden Unsicherheiten diskutiert. Die experimentellen Informationen und die quantitativen thermodynamischen Daten werden in einer generischen Fallstudie angewandt, um die Auswirkungen der verschiedenen geochemischen Lösungsbedingungen der verschiedenen Wirtsgesteinsysteme, die als HAW-Endlager in Deutschland in Frage kommen, auf die Löslichkeit und Speziation ausgewählter Radionuklide darzustellen. 